Supplementary information

\title{
Thermally-released arsenic in porewater from sediments in the Cold Lake area of Alberta, Canada
}

Muhammad Babar Javed and Tariq Siddique*

Department of Renewable Resources, University of Alberta, Edmonton, T6G 2G7, Canada

Corresponding author e-mail: tariq.siddique@ualberta.ca

The following supplementary information contains six tables (Table S1 to S6) and three figures (Figure S1 to S3) on 10 pages including this cover page. 
Supplementary information

Table S1. Particle size distribution of the ground sediments (S1-S7) determined using laser particle size analyzer.

$\begin{array}{llll}\text { Sample ID } & \text { Dx }(10)^{\mathrm{a}} & \begin{array}{c}\text { Dx (50) } \\ \mu \mathrm{m}\end{array} & \mathrm{Dx}(90)^{\mathrm{a}} \\ \text { S1 } & 7.2 & 52.8 & 120.8 \\ \text { S2 } & 3.8 & 25.5 & 118.0 \\ \text { S3 } & 6.4 & 39.7 & 101.7 \\ \text { S4 } & 5.0 & 32.3 & 112.0 \\ \text { S5 } & 6.1 & 35.8 & 117.4 \\ \text { S6 } & 7.7 & 65.9 & 114.9 \\ \text { S7 } & 7.8 & 27.5 & 89.6\end{array}$

${ }^{\mathrm{a}}$ Dx (10), Dx (50) and Dx (90) represent 10, 50 and 90\% particles, respectively that are less than the given sizes in the samples. 
Supplementary information

Table S2. A sequential extraction procedure used for As fractionation in the sediments.

\begin{tabular}{|c|c|c|}
\hline Fraction & Target Phase & Extractant $^{\mathrm{a}}$ \\
\hline $\mathrm{F} 1$ & Soluble As & DI water, $30 \mathrm{~min}$ \\
\hline F2 & Exchangeable As & $1 \mathrm{M} \mathrm{NaOAc}, \mathrm{pH} 8.2,2$ h \\
\hline F3 & Specifically adsorbed As & $\begin{array}{l}1 \mathrm{M} \mathrm{NaH}_{2} \mathrm{PO}_{4}, \mathrm{pH} 5,16 \text { and } \\
24 \mathrm{~h}\end{array}$ \\
\hline F4 & Carbonate bound As & $1 \mathrm{M} \mathrm{NaOAc}, \mathrm{pH}$ 5, 5 h \\
\hline F5 & $\begin{array}{l}\text { As co-precipitated with amorphous } \\
\text { Fe, Al and Mn oxyhydroxides }\end{array}$ & $\begin{array}{l}0.2 \text { M Ammonium oxalate, } \\
\text { pH 3, } 2 \text { h }\end{array}$ \\
\hline F6 & $\begin{array}{l}\text { As co-precipitated with crystalline } \\
\text { Fe, Al and Mn oxyhydroxides }\end{array}$ & $\begin{array}{l}0.05 \mathrm{M} \text { Ti-citrate-EDTA- } \\
\text { bicarbonate, } \mathrm{pH} \text { 7, } 2 \text { h twice }\end{array}$ \\
\hline F7 & $\begin{array}{l}\text { As associated with As oxides and } \\
\text { silicate clays }\end{array}$ & $10 \mathrm{M} \mathrm{HF}, 1$ and $24 \mathrm{~h}$ \\
\hline F8 & $\begin{array}{l}\text { As co-precipitated with pyrite and } \\
\text { orpiment }\end{array}$ & $16 \mathrm{~N} \mathrm{HNO}_{3}, 2 \mathrm{~h}$ twice \\
\hline F9 & $\begin{array}{l}\text { As associated with organic matter } \\
\text { and recalcitrant sulfides }\end{array}$ & $\begin{array}{l}30 \% \mathrm{H}_{2} \mathrm{O}_{2}+1 \mathrm{M} \mathrm{NH}_{4} \mathrm{OAc} \\
(1: 2), \mathrm{pH} 2,16 \mathrm{~h}\end{array}$ \\
\hline F10 & Residual As & $\begin{array}{l}\text { Concentrated } \mathrm{HNO}_{3} \text {, } \\
\text { microwave digestion }\end{array}$ \\
\hline
\end{tabular}

This information has been retrieved from Javed et al., $2013^{16}$

${ }^{\text {a }}$ Extractants were added to sediments in sequential order (F1-F10) and the supernatants were removed after extraction and centrifugation of each extraction. Sediments were washed with deionized water for 30 min after each extraction and the aqueous phase separated by centrifugation was pooled with the decanted supernatant of the preceding extractant. 
Table S3. Arsenic concentrations in different fractions after thermal treatment in sediments (S1S7) moistened with synthetic aquifer water at $15 \%$ water content.

\begin{tabular}{llllllll} 
Fraction & S1 & S2 & S3 & S4 & S5 & S6 & S7 \\
& & & \multicolumn{5}{c}{$\mu \mathrm{kg}^{-1}$} \\
F1 & $186 \pm 24$ & $281 \pm 28$ & $489 \pm 48$ & $889 \pm 64$ & $920 \pm 88$ & $1059 \pm 109$ & $570 \pm 63$ \\
F2 & $375 \pm 50$ & $482 \pm 48$ & $301 \pm 41$ & $872 \pm 72$ & $105 \pm 34$ & $674 \pm 57$ & $489 \pm 42$ \\
F3 & $573 \pm 61$ & $579 \pm 45$ & $1392 \pm 221$ & $1198 \pm 356$ & $1681 \pm 238$ & $5581 \pm 169$ & $3909 \pm 247$ \\
F4 & ND & ND & ND & ND & ND & ND & ND \\
F5 & $292 \pm 58$ & $291 \pm 38$ & $681 \pm 75$ & $675 \pm 118$ & $1207 \pm 107$ & $2457 \pm 386$ & $2360 \pm 254$ \\
F6 & $860 \pm 148$ & $1082 \pm 102$ & $1109 \pm 75$ & $1206 \pm 119$ & $2491 \pm 315$ & $3891 \pm 290$ & $6158 \pm 421$ \\
F7 & $186 \pm 48$ & $182 \pm 39$ & $1576 \pm 116$ & $1692 \pm 182$ & $1860 \pm 382$ & $3359 \pm 277$ & $2307 \pm 174$ \\
F8 & $281 \pm 65$ & $365 \pm 72$ & $1190 \pm 114$ & $1260 \pm 69$ & $2471 \pm 270$ & $2571 \pm 188$ & $8068 \pm 491$ \\
F9 & $\mathrm{ND}$ & $\mathrm{ND}$ & $\mathrm{ND}$ & $\mathrm{ND}$ & $\mathrm{ND}$ & $\mathrm{ND}$ & $2764 \pm 280$ \\
F10 & $\mathrm{ND}$ & $\mathrm{ND}$ & $\mathrm{ND}$ & $\mathrm{ND}$ & $\mathrm{ND}$ & $\mathrm{ND}$ & $\mathrm{ND}$
\end{tabular}

ND: not detected

The values in each fraction are means of six observations (duplicate sediment samples $\mathrm{x} 3$ subsamples from each sediment sample).

Legends (F1 - F10) represent different As fractions: F1, water soluble As; F2, ionically bound/exchangeable As; F3, strongly/specifically adsorbed As; F4, As bound in carbonates; F5, As coprecipitated with amorphous Fe, Al and Mn oxyhydroxides; F6, As coprecipitated with crystalline Fe, Al and Mn oxyhydroxides; F7, As associated with its oxides and silicate clays; F8, As coprecipitated with pyrite and orpiment; F9, As associated with organic matter and recalcitrant sulfides; and F10, residual As. 
Supplementary information

Table S4. Arsenic concentrations in different fractions after thermal treatment in sediments (S1S7) moistened with synthetic aquifer water at $50 \%$ water content.

\begin{tabular}{llllllll} 
Fraction & S1 & S2 & S3 & S4 & S5 & S6 & S7 \\
& & & \multicolumn{5}{c}{$\mu \mathrm{kg}^{-1}$} \\
F1 & $212 \pm 37$ & $320 \pm 32$ & $526 \pm 39$ & $910 \pm 79$ & $1138 \pm 73$ & $1103 \pm 136$ & $638 \pm 91$ \\
F2 & $438 \pm 68$ & $531 \pm 57$ & $339 \pm 30$ & $1061 \pm 109$ & $138 \pm 21$ & $786 \pm 69$ & $521 \pm 48$ \\
F3 & $648 \pm 52$ & $640 \pm 68$ & $1429 \pm 96$ & $2109 \pm 472$ & $1742 \pm 307$ & $5608 \pm 225$ & $3937 \pm 274$ \\
F4 & ND & ND & ND & ND & ND & ND & ND \\
F5 & $327 \pm 41$ & $309 \pm 29$ & $742 \pm 94$ & $761 \pm 85$ & $1250 \pm 132$ & $2539 \pm 275$ & $2418 \pm 317$ \\
F6 & $937 \pm 125$ & $1159 \pm 85$ & $1139 \pm 92$ & $1251 \pm 148$ & $2509 \pm 363$ & $3944 \pm 362$ & $6205 \pm 212$ \\
F7 & $206 \pm 72$ & $205 \pm 52$ & $1649 \pm 182$ & $1701 \pm 202$ & $2063 \pm 401$ & $3408 \pm 362$ & $2283 \pm 199$ \\
F8 & $326 \pm 81$ & $435 \pm 81$ & $1209 \pm 102$ & $1298 \pm 84$ & $2536 \pm 298$ & $2648 \pm 239$ & $8135 \pm 303$ \\
F9 & ND & ND & ND & ND & ND & ND & $2808 \pm 170$ \\
F10 & ND & ND & ND & ND & ND & ND & ND
\end{tabular}

ND: not detected

The values in each fraction are means of six observations (duplicate sediment samples x 3 subsamples from each sediment sample).

Legends (F1 - F10) represent different As fractions: F1, water soluble As; F2, ionically bound/exchangeable As; F3, strongly/specifically adsorbed As; F4, As bound in carbonates; F5, As coprecipitated with amorphous Fe, Al and Mn oxyhydroxides; F6, As coprecipitated with crystalline Fe, Al and Mn oxyhydroxides; F7, As associated with its oxides and silicate clays; F8, As coprecipitated with pyrite and orpiment; F9, As associated with organic matter and recalcitrant sulfides; and F10, residual As. 
Supplementary information

Table S5. Arsenic concentrations in different fractions after thermal treatment in sediments (S1S7) moistened with deionized water at $15 \%$ water content.

\begin{tabular}{llllllll} 
Fraction & S1 & S2 & S3 & \multicolumn{1}{l}{ S4 } & S5 & S6 & S7 \\
& & & \multicolumn{5}{c}{$\mu \mathrm{kg}^{-1}$} \\
F1 & $302+36$ & $288+24$ & $1172+76$ & $1295+68$ & $1669+106$ & $126+27$ & $743+46$ \\
F2 & $286+57$ & $516+48$ & $201+38$ & $625+31$ & ND & $577+76$ & $381+30$ \\
F3 & $588+48$ & $565+34$ & $1067+105$ & $2066+163$ & $1202+85$ & $5470+126$ & $3880+228$ \\
F4 & ND & ND & ND & ND & ND & ND & ND \\
F5 & $289+42$ & $309+19$ & $691+40$ & $686+97$ & $1084+138$ & $2359+268$ & $2185+164$ \\
F6 & $866+71$ & $1083+69$ & $1061+127$ & $1185+64$ & $1465+150$ & $3855+146$ & $6194+99$ \\
F7 & $197+31$ & $201+33$ & $1573+41$ & $1694+66$ & $1943+164$ & $3356+254$ & $2265+215$ \\
F8 & $334+40$ & $391+27$ & $1176+116$ & $1280+86$ & $2468+241$ & $2568+184$ & $8064+259$ \\
F9 & ND & ND & ND & ND & ND & ND & $2795+143$ \\
F10 & ND & ND & ND & ND & ND & ND & ND
\end{tabular}

ND: not detected

The values in each fraction are means of six observations (duplicate sediment samples $\mathrm{x} 3$ subsamples from each sediment sample).

Legends (F1 - F10) represent different As fractions: F1, water soluble As; F2, ionically bound/exchangeable As; F3, strongly/specifically adsorbed As; F4, As bound in carbonates; F5, As coprecipitated with amorphous Fe, Al and Mn oxyhydroxides; F6, As coprecipitated with crystalline Fe, Al and Mn oxyhydroxides; F7, As associated with its oxides and silicate clays; F8, As coprecipitated with pyrite and orpiment; F9, As associated with organic matter and recalcitrant sulfides; and F10, residual As. 
Supplementary information

Table S6. Arsenic concentrations in different fractions after thermal treatment in sediments (S1S7) moistened with deionized water at $50 \%$ water content.

\begin{tabular}{llllllll} 
Fraction & S1 & S2 & S3 & S4 & S5 & S6 & S7 \\
& & & \multicolumn{5}{c}{$\mu \mathrm{kg}^{-1}$} \\
F1 & $328+29$ & $315+31$ & $1227+59$ & $1309+73$ & $1758+183$ & $137+17$ & $784+52$ \\
F2 & $340+66$ & $542+56$ & $237+26$ & $651+48$ & ND & $649+58$ & $409+32$ \\
F3 & $629+53$ & $538+29$ & $1106+92$ & $2140+128$ & $1239+103$ & $5581+140$ & $3706+171$ \\
F4 & ND & ND & ND & ND & ND & ND & ND \\
F5 & $307+27$ & $327+24$ & $737+53$ & $744+82$ & $1129+146$ & $2396+209$ & $2224+190$ \\
F6 & $917+77$ & $1129+87$ & $941+84$ & $1209+77$ & $1544+126$ & $3896+131$ & $6222+76$ \\
F7 & $229+35$ & $226+27$ & $1608+50$ & $1733+79$ & $2060+201$ & $2450+277$ & $2290+177$ \\
F8 & $361+31$ & $416+33$ & $1198+88$ & $1307+72$ & $2494+183$ & $2594+140$ & $8142+231$ \\
F9 & ND & ND & ND & ND & ND & ND & $2839+162$ \\
F10 & ND & ND & ND & ND & ND & ND & ND
\end{tabular}

ND: not detected

The values in each fraction are means of six observations (duplicate sediment samples $\mathrm{x} 3$ subsamples from each sediment sample).

Legends (F1 - F10) represent different As fractions: F1, water soluble As; F2, ionically bound/exchangeable As; F3, strongly/specifically adsorbed As; F4, As bound in carbonates; F5, As coprecipitated with amorphous Fe, Al and Mn oxyhydroxides; F6, As coprecipitated with crystalline Fe, Al and Mn oxyhydroxides; F7, As associated with its oxides and silicate clays; F8, As coprecipitated with pyrite and orpiment; F9, As associated with organic matter and recalcitrant sulfides; and F10, residual As. 


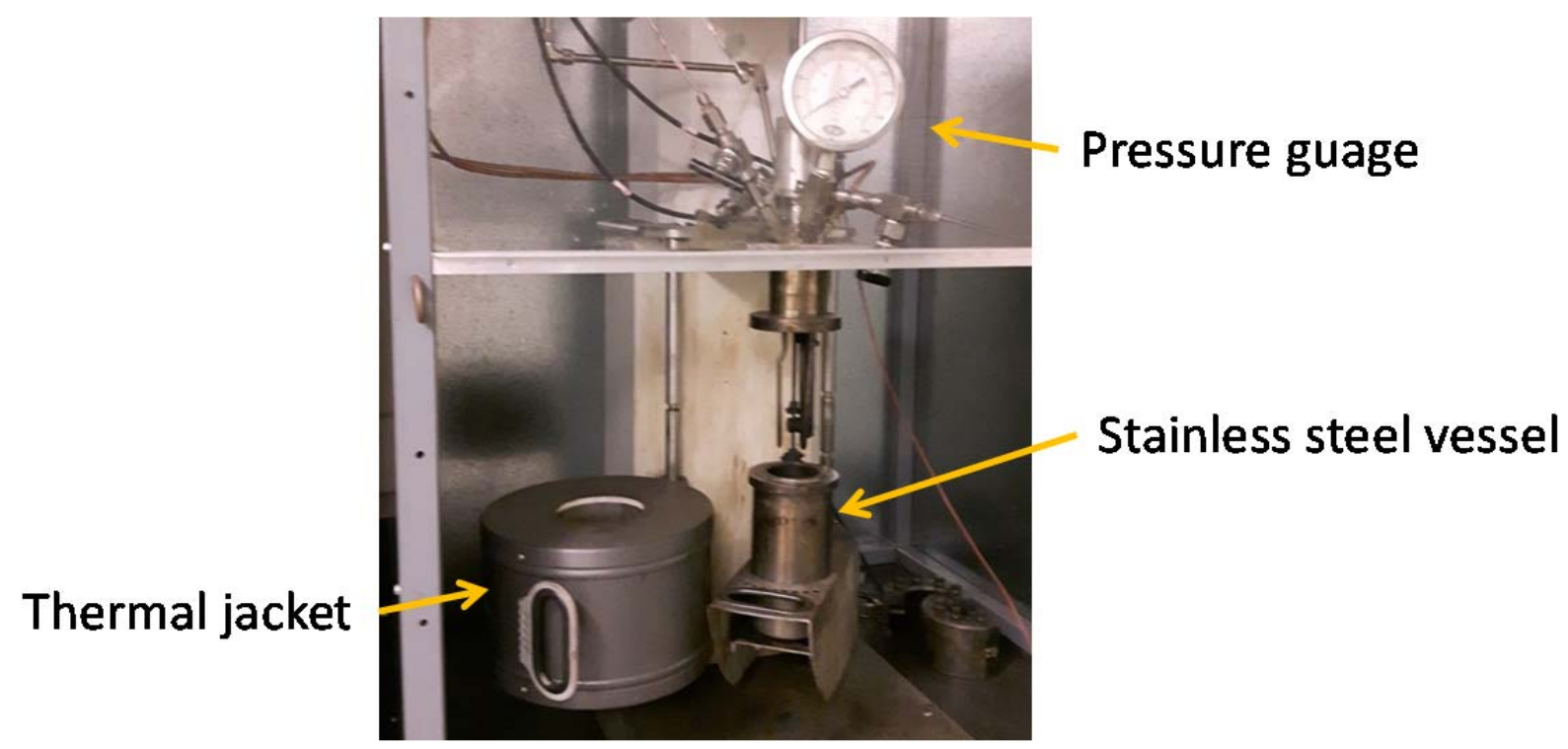

Figure S1. Autoclave batch reactor used for thermal treatment of sediments. 

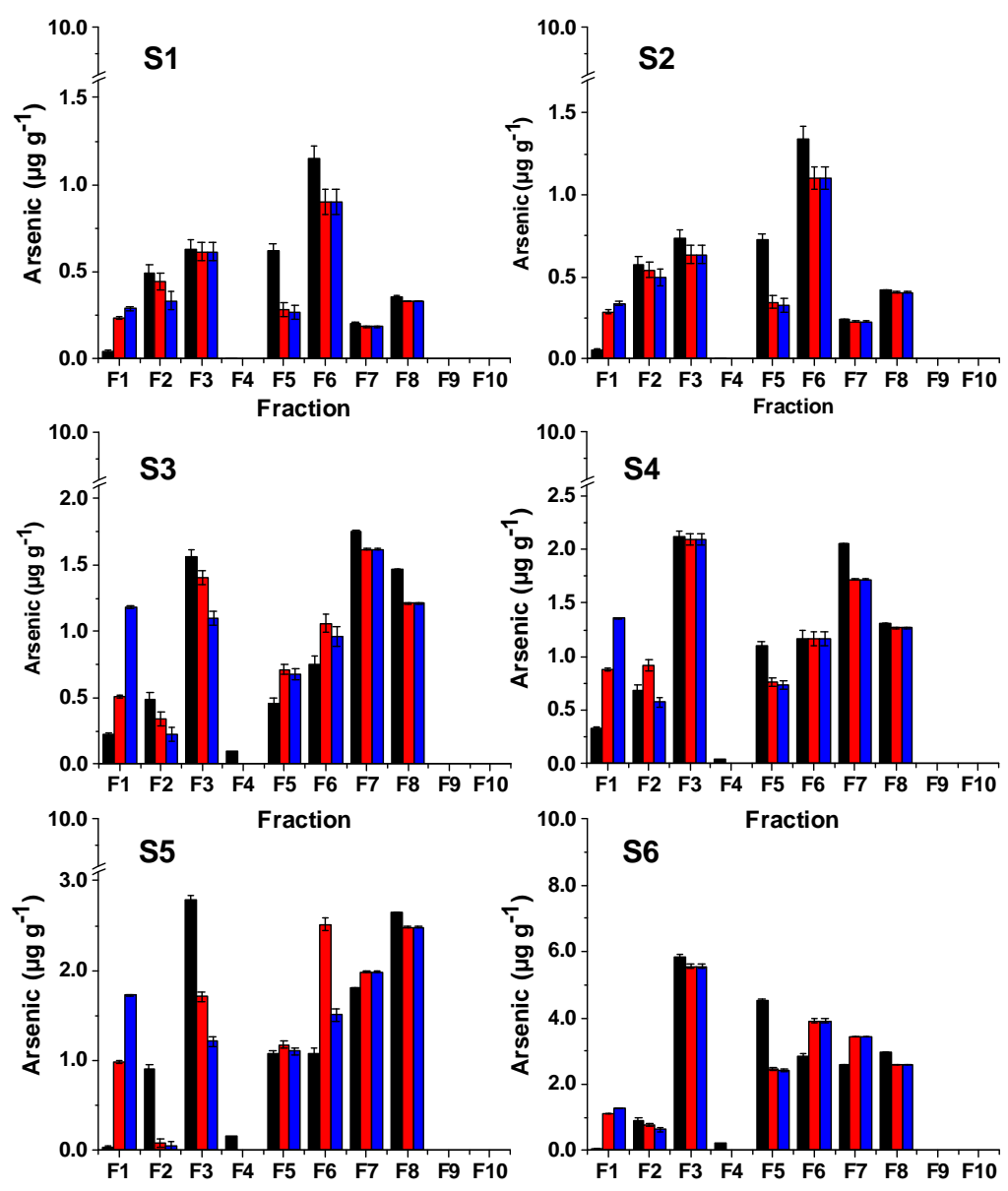

S6
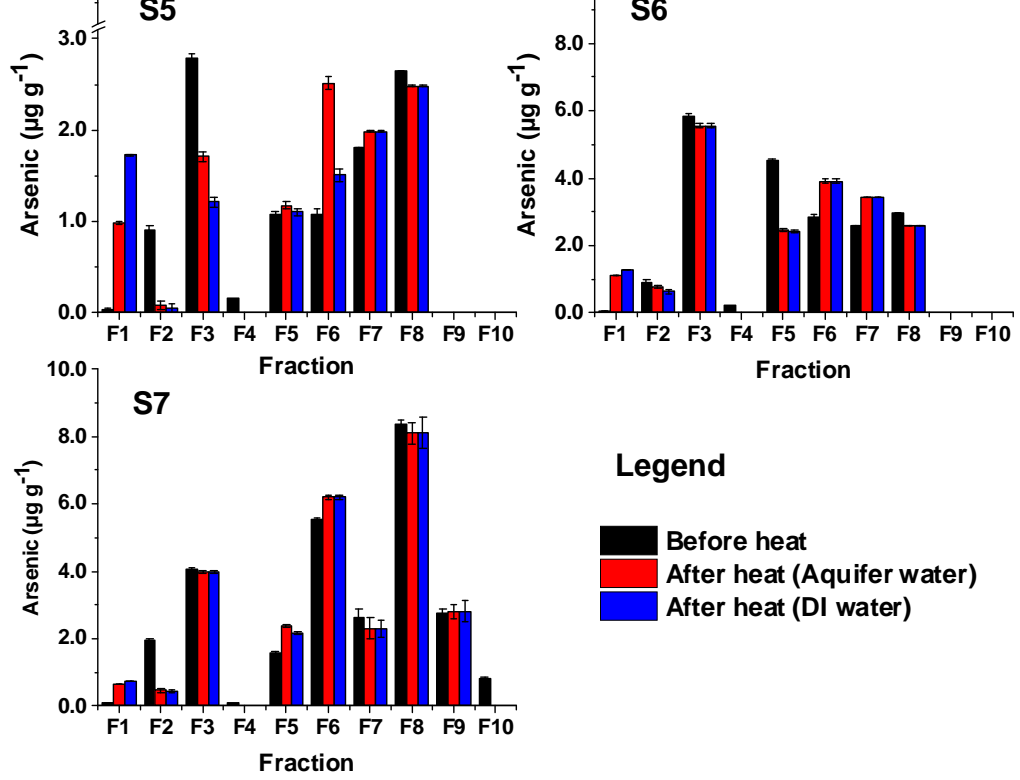

Legend

Before heat

After heat (Aquifer water)

After heat (DI water)

Figure S2. Effect of heat $\left(\sim 200^{\circ} \mathrm{C}\right)$ on arsenic (As) fractionation in sediments (S1-S7) moistened with synthetic aquifer (red bars) or deionized water (blue bars) at aquitard moisture level. The results obtained at 15 and 50\% water regimes (Tables S3- S6) were averaged to construct this figure. Values are the means of samples $(n=6$; duplicate sediment samples $\times 3$ subsamples from each sediment sample) and error bars, where visible, represent the standard deviation.

F1 to F10 on X-axis represent As fractions: F1, water soluble As; F2, ionically bound/exchangeable As; F3, strongly/specifically adsorbed As; F4, As bound in carbonates; F5, As coprecipitated with amorphous Fe, Al and Mn oxyhydroxides; F6, As coprecipitated with crystalline Fe, Al and Mn oxyhydroxides; F7, As associated with its oxides and silicate clays; F8, As coprecipitated with pyrite and orpiment; F9, As associated with organic matter and recalcitrant sulfides; and F10, residual As. 

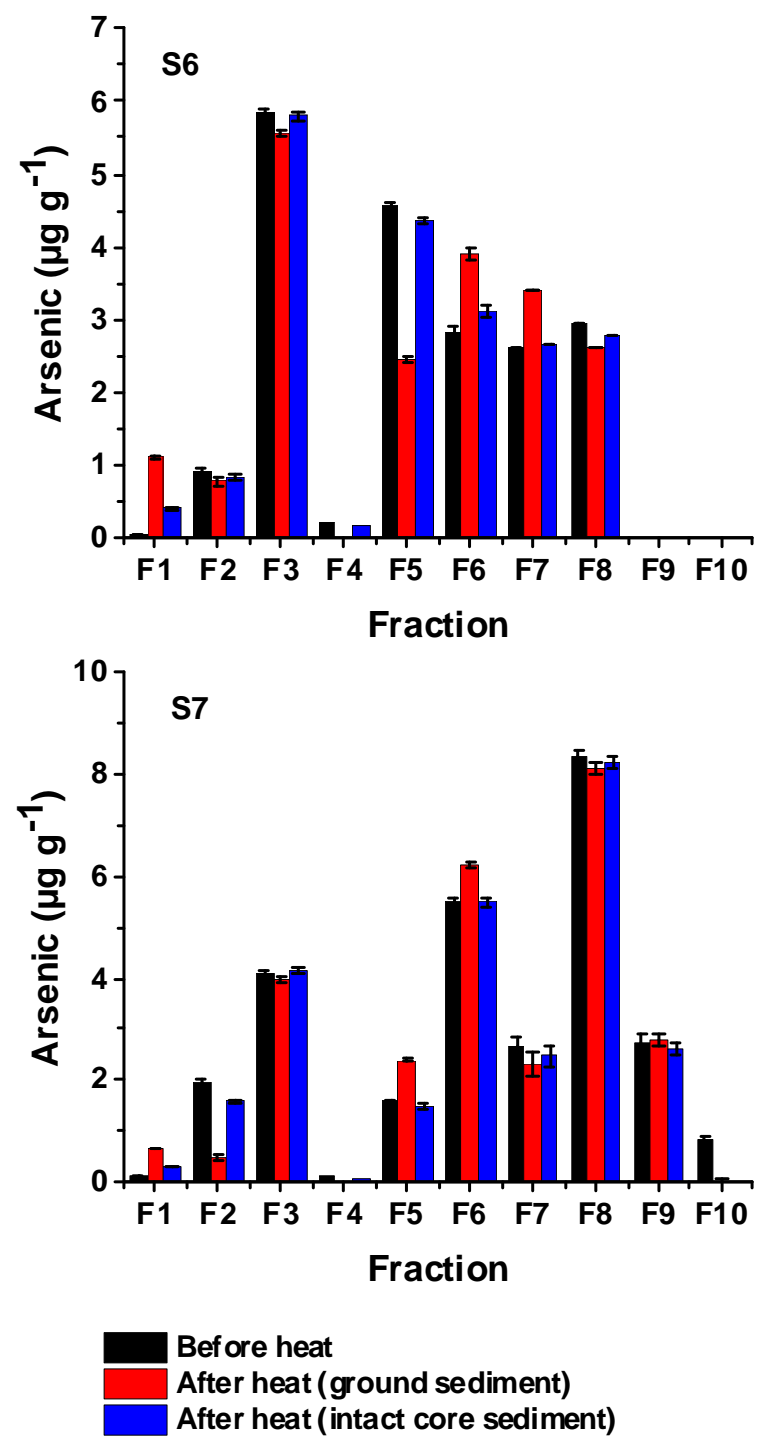

Figure S3. Effect of heat $\left(200^{\circ} \mathrm{C}\right)$ on arsenic (As) fractionation in ground versus intact core sediments (S6 and S7) moistened with synthetic aquifer water. Values are the means of triplicate samples and errors bars where visible represent the standard deviation. The intact core sediments were moistened with synthetic aquifer water to saturate the sediments ( 25\% moisture), and the results of As fractionation were compared to the average of the fractionation results obtained using ground sediments moistened with synthetic aquifer water at 15 and 50\%.

F1 to F10 on X-axis represent As fractions: F1, water soluble As; F2, ionically bound/exchangeable As; F3, strongly/specifically adsorbed As; F4, As bound in carbonates; F5, As coprecipitated with amorphous Fe, Al and Mn oxyhydroxides; F6, As coprecipitated with crystalline Fe, Al and Mn oxyhydroxides; F7, As associated with its oxides and silicate clays; F8, As coprecipitated with pyrite and orpiment; F9, As associated with organic matter and recalcitrant sulfides; and F10, residual As. 